\section{P3.276 NEISSERIA GONORRHOEAE (GC) RESISTANCE SURVEILLANCE IN SELECTED POPULATIONS OF FIVE COUNTRIES}

doi:10.1136/sextrans-2013-051184.0732

${ }^{1,2}$ A Y Tsai, ${ }^{3,4} \mathrm{E}$ Dueger, ${ }^{5} \mathrm{G}$ E Macalino, ${ }^{6} \mathrm{~S} M$ Montano, ${ }^{7} \mathrm{M}$ Mbuchi, ${ }^{8} \mathrm{~N}$ Puplampu,

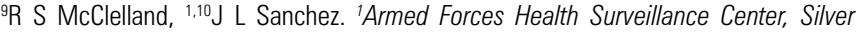
Spring, MD, United States; ${ }^{2}$ Oak Ridge Institute for Science and Education Postgraduate Research Participation Program, US Army Public Health Command, Aberdeen Proving Ground, MD, United States; ${ }^{3}$ US Naval Medical Research Unit No. 3, Cairo, Egypt; ${ }^{4}$ Global Disease Detection Branch, US Centers for Disease Control and Prevention (CDC), Atlanta, GA, United States; ${ }^{5} /$ fectious Disease Clinical Research Program (IDCRP), Uniformed Services University, Bethesda, MD, United States; ${ }^{6}$ US Naval Medical Research Unit No. 6, Lima, Peru; 'US Army Medical Research Unit-Kenya, Nairobi, Kenya; ${ }^{8}$ US Naval Medical Research Unit No. 3 Detachment, Accra, Ghana; ${ }^{9}$ University of Washington, Seattle, WA, United States; ${ }^{10} \mathrm{Henry}$ M. Jackson Foundation for the Advancement of Military Medicine, Inc., Bethesda, MD, United States

Background GC constitutes the second most commonly reportable disease in the United States with over 320,000 cases annually. With the emergence of drug-resistant GC in the past 40 years, treatment options have become very limited. Hence, the U.S. Department of Defense has launched a GC resistance surveillance network in 8 countries; preliminary results are reported from the United States, Djibouti, Ghana, Kenya, and Peru.

Methods Patients with urethritis, cervicitis or vaginitis symptoms were recruited at participating clinics serving military personnel and beneficiaries, civilians, and at-risk groups of men who have sex with men and female commercial sex workers. Urethral swabs were collected from men; urethral or vaginal swabs from women; diagnosis was done using culture identification, nucleic acid amplification testing, and real-time PCR. Antimicrobial susceptibility testing (AST) was conducted on GC positive isolates using real-time PCR, disc diffusion, and E-test strip methods.

Results Overall, 108 (6\%) of 1,694 enrolled subjects tested positive for GC. Prevalence was found to be highest in Kenya where 33 (38\%) of 86 patients were positive and was lowest in Peru where only $30(2 \%)$ of 1,296 patients were positive. AST results were available on 66 GC positives; resistance to at least three antibiotics was observed across all overseas sites. Greatest variability in resistance was noted in Djibouti as follows: penicillin $(100 \%)$, tetracycline $(88 \%)$, ciprofloxacin (38\%), levofloxacin (17\%), cefepime (13\%), and ceftriaxone $(13 \%)$. High-level resistance $(100 \%)$ was also noted in Ghana to ciprofloxacin, penicillin, and tetracycline.

Conclusion These findings provide evidence of emerging drugresistant GC in several regions of the world; the resistance found against third-generation cephalosporin in Djibouti is especially noteworthy. With continuing global vigilance, GC drug resistance information will provide an important basis for the development of effective control measures, particularly among deployable forces and at-risk populations in geographical regions of military relevance.

\section{P3.277 DIAGNOSIS AND ANTIMICROBIAL RESISTANCE OF NEISSERIA GONORRHOEAE IN ESTONIA}

doi:10.1136/sextrans-2013-051184.0733

T Brilene, ${ }^{2} \mathrm{~A}$ Pöder, Eastern European Network for SexualReproductive Health. ${ }^{1}$ Department of Microbiology, Tartu University, Tartu, Estonia; ${ }^{2}$ Dermatology Clinic, Tartu University, Tartu, Estonia

Background The incidence of gonococcal infections in Estonia peaked in 1993 with an infection rate of 233 cases/100,000 population and declined to 16.2 cases/100,000 population in 2012. The greatest part of clinical laboratories desists from $N$. gonorrhoeae cultivation. $100 \%$ of Neisseria gonorrhoeae diagnosis in Estonia is made by DNA detection techniques. Different tests are in use: Rapid
DNA probe GEN-PROBE PACE (Gen-Probe Incorporated San Diego. USA), PCR with in house made primers other commercially available STI tests.

Aims to improve laboratory diagnosis of gonorrhoe and monitoring of antimicrobial susceptibilities of $N$ gonorrhoeae to investigate treatment failure and to evaluate the efficacy of currently recommended therapies.

Methods In 2007, Estonian IUSTI branch has elaborated National Guidelines for the STI management (on the base of the European STD Guidelines and Eastern European Network for Sexual and Reproductive Health) with it's second revission in 2011. For N.gonorrhoeae diagnosis was recommended to start with molecular test and then if positive to continue with cultural method, using disc diffusion method and E-tests for AMR. WHO reference panel N.gonorrhoeae was obtained from reference laboratory in Örebro, Sweden.

Results Totally 24 isolates obtained. Gonococci (14) were collected from urethral swabs of men, 8 strains were isolated from female cervical swabs, gender was unknown for 2 cases (anonymous). AMR detected in 4 isolates; 2 were strains isolated after treatment failure: one - resistant to Pen, Tetra, Cipro and susceptible to Ceftriaxone; second - resistant to Pen, Tetra, Cipro and had decreased susceptibility (resistance) to Ceftriaxone (MIC $0.25 \mathrm{mg} / \mathrm{L}$ ). In addition in 2 isolates MICs to Ceftriaxone were $0.38 \mathrm{mg} / \mathrm{L}$ and $0.50 \mathrm{mg} / \mathrm{L}$. AMR were detected to penicillin (12.5\%), to ciprofloxacin (8.4\%) and to tetracycline $(8.4 \%)$.

Conclusion Gonorrhoea may become untreatable under certain circumstances and surveillance of $N$. gonorrhoeae AMR is crucial in Estonia.

\section{P3.278 AZITHROMYCIN SUSCEPTIBILITIES IN CANADIAN NEISSERIA GONORRHOEAE ISOLATES (2010-2011)}

doi:10.1136/sextrans-2013-051184.0734

II Martin, 'P Sawatzky, 'G Liu, ${ }^{2} \mathrm{~B}$ Lefebvre, ${ }^{3} \mathrm{M}$ Lovgren, ${ }^{4} \mathrm{~V}$ Allen, ${ }^{5} \mathrm{~L}$ Hoang, ${ }^{6} \mathrm{G}$ Horsman, 'T Wong. 'Public Health Agency of Canada, National Microbiology Laboratory, Winnipeg, MB, Canada; 'Laboratoire de sante publique du Quebec, Ste-Anne-deBellevue, QC, Canada; ${ }^{3}$ Provincial Laboratory for Public Health Alberta, Edmonton, AB, Canada; ${ }^{4}$ Public Health Ontario Laboratories, Toronto, ON, Canada; ${ }^{5}$ British Columbia Centres for Disease Control, Vancouver, BC, Canada; ${ }^{6}$ Saskatchewan Disease Control Laboratory, Regina, SK, Canada; 'Public Health Agency of Canada, Ottawa, ON, Canada

Background Neisseria gonorhoeae have developed resistance to many antibiotics and current Canadian STI guidelines recommend azithromycin as part of a combination therapy for gonorrhoea.

Methods Between 2010 and 2011, N. gonorrhoeae strains were isolated or collected by Canadian provincial public health laboratories and submitted to the National Microbiology Laboratory (NML) $(\mathrm{N}=2392)$. Isolates are submitted to the NML only when the provincial laboratories identify resistance to at least one antibiotic or if the provincial laboratories do not conduct any antimicrobial susceptibility testing. Minimum inhibitory concentrations (MICs) were determined by agar dilution and the $N$. gonorrhoeae multi-antigen sequence typing (NG-MAST) was used for molecular typing. Possible mutations in the mtrR gene and promoter region, the $23 \mathrm{~S}$ rRNA (4 alleles) and $\mathrm{L} 22$ riboprotein genes were determined by sequencing.

Results Azithromycin resistance was 3.0\% in 2010 (37/1233) and $1.1 \%$ in $2011(13 / 1158)$ amongst all the isolates tested at NML. Forty-five azithromycin resistant isolates (MICs ranging from 2 to $\geq$ $256 \mu \mathrm{g} / \mathrm{ml}$ ) were characterised. The A deletion in the $m t r R$ efflux pump were identified in $37.8 \%(\mathrm{n}=17)$ isolates. Additional $m t r R$ mutations include: A39T $(28.9 \%, \mathrm{n}=13)$ and G45D $(4.4 \%, \mathrm{n}=2)$. One isolate was identified with mutation D89A in the L22 riboprotein. Thirty-three isolates (73.3\%) were identified with the C2599T mutation in at least 1 of the 4 alleles of the 23S rRNA. The A2143G 
mutation was identified in all 4 alleles in the two isolates with azithromycin MICs of $>=256 \mu \mathrm{g} / \mathrm{ml}$.

Conclusions With reducing susceptibility of cephalosporins as treatment for gonorrhoea, azithromycin may be relied on more to treat gonorrhoea. Rapid molecular detection of antimicrobial susceptibilities in N. gonorrhoeae is important to prevent the spread of untreatable multidrug resistant gonorrhoea.

\section{P3.279 FROM THE GROUND UP: BUILDING NATIONAL SURVEILLANCE OF ANTIMICROBIAL RESISTANCE IN NEISSERIA GONORRHOEAE IN CANADA}

\author{
doi:10.1136/sextrans-2013-051184.0735
}

'S L Totten, ${ }^{2}$ I Martin, ' $\mathbf{J}$ Njihia, 'T Atwood, 'C Archibald. 'Public Health Agency of Canada, Ottawa, ON, Canada, ${ }^{2}$ Public Health Agency of Canada, Winnipeg, MB, Canada

Background While there is evidence that gonococcal antimicrobial resistance (GC AMR) is increasing in parts of Canada, a national, standardised surveillance system does not currently exist to confirm these suspicions or identify the risks associated with acquiring a resistant GC infection.

Methods Currently, laboratory-based surveillance of GC AMR is standard practise for all positive gonorrhoea isolates tested by culture in Canada. Nine out of 13 provinces/territories employ culture for a proportion of the total gonorrhoea tests done in their jurisdictions (typically conducted by local/regional laboratories). Variation in methods at the provincial/territorial (P/T) level and limited epidemiologic data on resistant GC isolates limits national level surveillance.

To address gaps in current systems, a national protocol for GC AMR has been developed and approved by the Health CanadaPublic Health Agency of Canada Research Ethics Board, and recruitment of $\mathrm{P} / \mathrm{T}$ health authorities is in progress. Due to $\mathrm{P} / \mathrm{T}$ variations in public health legislation and health care practises, recruitment has necessitated innovative solutions to address the individual needs of jurisdictions while ensuring the coherence and comparability of the resulting data.

Results In 2011, the proportion of GC isolates resistant to azithromycin, penicillin, erythromycin, ciprofloxacin and tetracycline was $0.4 \%, 22.2 \%, 26.6 \%, 29.3 \%$, and $29.4 \%$, respectively. Enhanced surveillance in two jurisdictions is expected to commence in 2013. Although slightly different mechanisms are being used to address provincial needs, efforts are being made to ensure that resulting data are consistent and adhere to the national protocol.

Conclusion In Canada, surveillance of GC AMR is challenged by variations in practise and legislation at the $\mathrm{P} / \mathrm{T}$ level and competing priorities at all levels of government. Through collaboration with public health partners, progress is being made in obtaining data for analysis of national-level trends to assess risk factors associated with GC AMR and guide treatment recommendations.

\section{P3.280 GENTAMICIN SUSCEPTIBILITY PROFILE OF NEISSERIA GONORRHOEAE CLINICAL STRAINS ISOLATED IN BILBAO (SPAIN) DURING 2011 AND 2012}

doi:10.1136/sextrans-2013-051184.0736

M Nieto, G Ezpeleta, V Esteban, J Alava, M Camara, J Lopez de Munain, R Cisterna. Basurto University Hospital, Bilbao, Spain

Background Antimicrobial therapy is essential to control Neisseria gonorrhoeae (NG). The appearance of isolates with decreased susceptibility or resistant to third-generation cephalosporins (TGC) has raised several concerns. Recent clinical trials suggest that gentamicin could be an alternative for NG treatment, but no susceptibility data to this drug is available in European NG isolates. Objectives were: describe the epidemiological and antibiotic susceptibility profile to NG strains isolated.
Methods Descriptive retrospective study of NG strains isolated during 2011 and 2012. The susceptibility to cefixime, ceftriaxone, gentamicin and ciprofloxacin was performed using agar dilution (AD) and disc diffusion (DD) methods according to the CLSI guidelines.

Results A total of 275 NG strains were isolated: 143 urethral (52\%), 47 rectal (17\%), 44 pharyngeal (16\%), 28 endocervical (10\%), vaginal and $13(5 \%)$, belonging to 225 patients (174 men (77.3\%), 51 $(22.7 \%)$ women). The average age was 33.32 years ( $15-58$ years). About $80 \%$ of the samples (221) were from STD Clinics Infectious Service. 151 patients (67.1\%) were symptomatic at the moment of diagnosis, 32 cases (14.2\%) were detected during screening and 42 cases $(18.7 \%)$ during contact tracing. Urethritis was the most common clinical manifestation in men and accounted $71,3 \%$ of the cases.

All strains were susceptible to TGC by both methods. Ciprofloxacin showed $59.3 \%$ resistant strains by $\mathrm{AD}$ with low minor discordances between the two methods in 22 strains. $74.5 \%$ of the strains showed a MIC of $8 \mu \mathrm{g} / \mathrm{ml}$ to gentamicin (range: $2-16 \mu \mathrm{g} / \mathrm{ml}$ ), and inhibition zone in the DD method between 16 and $38 \mathrm{~mm}$.

Conclusions During the study period we found no resistant strains to TGC, but it was recorded a high rate of resistance to ciprofloxacin. The susceptibility profile of gentamicin is similar to those published but further research is needed to establish clinical breakpoints and doing a treatment recommendation.

\section{P3.281 GONORRHOEA ON THE RISE IN LATVIA - DIAGNOSTICS AND ANTIMICROBIAL RESISTANCE SURVEILLANCE}

doi:10.1136/sextrans-2013-051184.0737

'D Ozolins, ' $\mathrm{A}$ Zilevica, ${ }^{2} \mathrm{~V}$ Mavcutko, ${ }^{3} \mathrm{M}$ Unemo, ${ }^{4} \mathrm{M}$ Domeika, Eastern European Network for SexualReproductive Health. ${ }^{1}$ Faculty of Medicine, University of Latvia, Riga, Latvia; ${ }^{2}$ The Centre for Disease Prevention and Control and Infectology, Riga, Latvia; ${ }^{3}$ WHO Collaborating Centre for Gonorrhoea and other STIs, Örebro, Sweden, Orebro, Sweden; ${ }^{4}$ Department of Control and Prevention of Communicable Diseases Uppsala County Council, Uppsala, Eastern European Network for Sexual and Reproductive Health, Uppsala, Sweden

Background The emergence and international spread of antimicrobial resistance (AMR) in Neisseria gonorrhoeae has compromised the treatment and public health management of gonococcal infections. Appropriate diagnostics and surveillance of AMR are critical for controlling infections and in guiding international and national treatment guidelines to ensure appropriate patient management.

Methods A questionnaire-based survey regarding the diagnostics and AMR surveillance of N. gonorrhoeae in Latvia was conducted by the Centre for Disease Prevention and Control and Infectology of Latvia. Telephone interviews were carried out among the 73 biggest medical laboratories in Latvia.

Results In 2011, a total of 550 cases of gonorrhoea were reported giving a rate of 26.7 per 100000 population. In 2012 - 602 cases and an incidence of 29.5. In total, the incidence of gonorrhoea increased by $73.5 \%$ from 2010 to 2012 . For detection of N. gonorrhoeae, currently three laboratories are using nucleic acid amplification tests (NAATs), 10 - RNA probe hybridization tests, four laboratories culture, and 17 - Gram staining of specimen smears only. Monitoring of AMR in N. gonorrhoeae was initiated in 2010, however, during 2010 and 2012 in total only 35 isolates were tested. All 35 isolates were fully susceptible to cefixime, ceftriaxone, azithromycin, and spectinomycin, and the minimum inhibitory concentrations (MICs) of gentamicin were low. Resistance to ciprofloxacin was displayed by $43 \%$ of isolates, to penicillin by $31 \%$, and to tetracycline by $23 \%$. Conclusion The gonorrhoea incidence has significantly increased in Latvia during the recent years, which cannot only be explained by introduction of more sensitive diagnostics such as NAATs in a few laboratories. It is imperative to substantially strengthen the diagnostics and AMR surveillance in Latvia. 